\title{
Readmission rates of patients with COVID-19 after hospital discharge
}

\author{
Recep Alanli* (D), Murat Bulent Kucukay ${ }^{1}$, Kadir Serkan Yalcin
}

\section{SUMMARY}

OBJECTIVE: The purpose of this study was to inspect return and readmission reasons and rates of discharged patients with coronavirus disease 2019 (COVID-19).

METHODS: This is an observational descriptive retrospective study that was conducted with patients who had confirmed COVID-19 diagnosed with severe respiratory syndrome coronavirus-2 (SARS-CoV-2) polymerase chain reaction (PCR) and hospitalized between April 2020 and June 2021 in a tertiary care university hospital. Patients returning to the hospital after treatment for COVID-19, with symptoms related to COVID-19 within 30 days, were included. Patients under 18 years of age and who were hospitalized in the intensive care unit were excluded.

RESULTS: It was determined that of 369 discharged patients, 87 (23.5\%) returned to the hospital, 9 (2.4\%) were readmitted, and 1 $(0.02 \%)$ was deceased within 30 days. The most frequent reasons for returning to the hospital were dyspnea and cough complaints. Existence of pneumonia at first admission, levels of aspartate aminotransferase, lactate dehydrogenase, C-reactive protein, D-dimer, neutrophil counts, lymphocyte counts, and neutrophil-to-lymphocyte count ratios were found to be higher in patients who returned to hospital, compared with the patients who did not return.

CONCLUSIONS: Return rate of patients to hospital after discharge with COVID-19 was comparatively high, but readmissions to hospital and mortality rate were low. Comparatively, the higher rate of return to hospital within 30 days of discharge was thought to be resulting from prolonged signs and symptoms related to COVID-19. Since COVID-19 is a new and enigmatic disease and its long-term effects still need to be elucidated, long-term follow-ups of discharged patients will be adequate.

KEYWORDS: COVID-19. Patient readmission. 30-day readmission.

\section{INTRODUCTION}

The coronavirus disease 2019 (COVID-19) is a viral infection caused by subacute severe respiratory syndrome coronavirus- 2 (SARS-CoV-2), which spreads by air as respiratory droplets and may result in severe pneumonia and respiratory failure. After the first case in Wuhan, China, in December 2019, the disease has become pandemic in a very short time. It has been first reported in March 2020 in Turkey, and up to now, more than 5 million and 400 thousand patients and 50 thousand deaths have been reported ${ }^{1}$.
Symptoms and signs related to COVID-19 may persist for a long time. Information about the readmission rate of patients with COVID-19 is insufficient ${ }^{2}$. In two previous studies, readmission rates to the hospital for symptoms related to COVID-19 after discharge were 1.9 and $29.4 \%$, respectively ${ }^{3,4}$. There are very few studies inspecting readmissions of patients with COVID-19 in Turkey. One study reports a readmission rate of $7.1 \%$ within 30 days of discharge ${ }^{5}$. Detailed information about the clinical course and follow-up in the convalescence period after discharge may be

'Lokman Hekim University, Faculty of Medicine, Ankara Hospital, Department of Internal Diseases - Ankara, Turkey.

*Corresponding author: recepalanli@gmail.com

Conflicts of interest: the authors declare there is no conflicts of interest. Funding: none.

Received on August 19, 2021. Accepted on August 19, 2021. 
beneficial for the management of the course. In addition, if characteristics and clinical findings of patients whose clinical course has worsened in the convalescence period can be determined better; estimating the best timing for hospital discharge and issues that must be considered in the follow-up period may be more reliable and convenient for those patients 6 .

In the inspection of readmissions to the hospital after treatment for COVID-19, periods of 14-60 days were considered in different reports ${ }^{7,8}$. A follow-up period of 30 days after discharge is a reliable time to evaluate the development of unwanted outcomes related to COVID- $19^{3}$. Rate of readmission is strongly related to the existence of concomitant diseases, course, severity, and recovery rate of COVID-19 disease. Higher rates of readmission are related to bad prognosis for these patients ${ }^{2}$.

The aim of this study was to inspect reasons for returning or readmissions to the hospital and return, readmission rates, and mortality rates of patients with COVID-19 within 30 days of discharge.

\section{METHODS}

\section{Study design and population}

This is an observational descriptive retrospective study that was conducted with patients who had confirmed COVID-19 diagnosed and hospitalized between April 2020 and June 2021 in the internal medicine department of a tertiary care university hospital. Diagnosis, hospitalization criteria, treatment, and discharge decisions were made according to the guidelines of the Scientific Advisory Committee of the National Ministry of Health?.

This study was approved by the Local Ethics Committee (approval no: 2021/9). This study was conducted in compliance with Helsinki Declaration and Good Clinical Practice Directives.

\section{Inclusion criteria}

Patients who were hospitalized and discharged after treatment for COVID-19 disease and who had positive SARS-CoV-2 polymerase chain reaction (PCR) test results were included.

\section{Exclusion criteria}

Patients below 18 years of age, patients who were hospitalized in the intensive care unit, and patients who had negative PCR test results were excluded. Patients who returned to the hospital for previously planned admissions or symptoms unrelated to COVID-19 were excluded.

\section{COVID-19 diagnostic criteria}

COVID-19 diagnosis was confirmed with the detection of specific viral RNA sequences in rRT-PCR by nucleic acid amplification test.

\section{Study setting}

This study was conducted in a tertiary university hospital with 250 beds located in the capital city of Turkey.

\section{Data collection}

Patients were inspected for returning to a health care facility (either the hospital in which treatments for COVID-19 were given or admissions to other hospitals) for COVID-19-related signs and symptoms within 30 days of discharge. Hospital records of patients were inspected, and patients who had missing information were confirmed by phone calls.

Demographic characteristics, laboratory test results, radiologic imaging study results, treatment regiments for COVID19 , length of hospitalization, and accompanying diseases were recorded. Symptoms, readmission rates, and mortality rates of patients within 30 days of discharge were recorded. Only first readmission data were considered. Data of patients with readmissions and without readmissions were compared.

Complete blood count (i.e., lymphocyte, neutrophil, thrombocyte counts), alanine aminotransferase (ALT), aspartate aminotransferase (AST), creatinine, lactic dehydrogenase (LDH), C-reactive protein (CRP), ferritin, D-dimer, and thorax computed tomography (CT) data were inspected. Neutrophil-tolymphocyte ratio (NLR) was calculated by the division of neutrophil counts to lymphocyte counts. Complete blood counts were analyzed by Sysmex XN-1000 (USA) as 22 parameters. ALT, AST, creatinine, LDH, D-dimer, and CRP tests were analyzed by Roche Hitachi Cobas 501 (Switzerland). Ferritin levels were analyzed by Roche Hitachi Cobas 601 (Switzerland).

\section{Statistical analysis}

Data were analyzed using SPSS version 25.0 statistical package (Armonk, NY, USA). Data distributions were evaluated by Shapiro-Wilk test. Data were presented as mean \pm standard deviation for normally distributed variables and as median, interquartile range, and minimum-maximum for non-normally distributed variables. Comparisons between groups in normally distributed variables were done by independent samples $t$ test and Mann-Whitney $U$ test for not normally distributed variables. The chi-square test was used for the comparison of qualitative data. The Fisher's exact test was used when the chi-square test cannot be used. Logistic regression analysis and odds ratios were determined. A $p<0.05$ was considered statistically significant. 


\section{RESULTS}

A total of 437 patients were hospitalized with COVID-19 in the study period at the study hospital. Of these, $22(5 \%)$ died in the hospitalization period, $5(1.1 \%)$ were transferred to other hospitals for various reasons, and 41 (9.4\%) patients could not be reached after discharge from the hospital, and these 68 patients were excluded. The remaining 369 (84.4\%) patients were included in this study.

Of the 369 patients, 182 were males (49.3\%) and 187 $(50.7 \%)$ were females. The mean age was $52.52 \pm 17.90$ years (males $54.05 \pm 16.73$ and females $51.02 \pm 18.90$ ). The mean hospitalization period of patients at first admission to the hospital for COVID-19 was $6.10 \pm 4.18$ days. The mean time for returning to the hospital after discharge was $9.16 \pm 7.40$ days. Notably, 95 (25.7\%) patients were above 65 years of age, and $274(74.3 \%)$ patients were below 65 years of age.

Of the 369 discharged patients, $87(23.5 \%)$ returned to the hospital for symptoms related to COVID-19. Nine (2.4\%) of these patients were readmitted to the hospital and only one $(0.2 \%)$ died. Returning reasons of discharged patients were shortness of breath in $68(78.1 \%)$, cough in $45(51.7 \%)$, arthralgia in 37 (42.5\%), chest pain in 37 (42.5\%), headache in $20(22.9 \%)$, high fever in $16(18.3 \%)$, and change/loss of sense of taste or smell in 13 (14.9\%).

Demographic characteristics, concomitant diseases, and antiviral treatments of patients with COVID-19 with or without 30-day readmission to the hospital were shown in Table 1. Hydroxychloroquine was used in the treatment of $80(21.6 \%)$ patients, favipiravir was used in 215 (58.3\%) patients, and both drugs were used in $74(20.1 \%)$ patients. At first admission to the hospital, 279 (75.6\%) patients had pneumonia.

Laboratory test results of patients with COVID-19 with or without 30-day readmission to the hospital were shown in Table 2.

To understand factors affecting patients' return to the hospital, logistic regression analysis was performed. The existence of pneumonia at first hospitalization was associated with a 2.81 times increase in the risk of readmission, and the existence of concomitant malignancy was associated with a 3.45 times increase in the risk of readmission. Logistic regression analysis results for readmission of patients with COVID-19 are shown in Table 3.

Table 1. Demographic characteristics and antiviral treatments of patients with COVID-19 with or without 30-day readmission to hospital.

\begin{tabular}{l|c|c|c|c}
\hline Property & $\begin{array}{c}\text { Total participants } \\
(\mathrm{n}=369)\end{array}$ & $\begin{array}{c}\text { With readmission } \\
(\mathrm{n}=87)\end{array}$ & $\begin{array}{c}\text { Without readmission } \\
(\mathrm{n}=282)\end{array}$ & $\mathrm{p}$-value \\
\hline Male/female & $182 / 187$ & $48 / 39$ & $134 / 148$ & 0.212 \\
\hline Age & $52.52 \pm 17.90$ & $53.01 \pm 19.21$ & $52.37 \pm 17.51$ & 0.769 \\
\hline Length of hospitalization* & $5(\mathrm{IQR}=4-7)$ & $6(\mathrm{IQR}=4-9)$ & $5(\mathrm{IQR}=3-7)$ & $\mathbf{0 . 0 4 4}$ \\
\hline Age above 65 years, $\mathrm{n}(\%)$ & $95(25.7)$ & $25(28.7)$ & $70(24.8)$ & 0.466 \\
\hline Hypertension, $\mathrm{n}(\%)$ & $109(9.5)$ & $30(34.4)$ & $79(28)$ & 0.248 \\
\hline Diabetes mellitus, $\mathrm{n}(\%)$ & $69(18.6)$ & $17(19.5)$ & $52(18.4)$ & 0.818 \\
\hline Coronary artery disease, $\mathrm{n}(\%)$ & $67(18.1)$ & $21(24.1)$ & $46(16.3)$ & 0.098 \\
\hline $\begin{array}{l}\text { Chronic obstructive pulmonary } \\
\text { disease, } \mathrm{n}(\%)\end{array}$ & $42(11.3)$ & $12(13.7)$ & $30(10.6)$ & 0.418 \\
\hline Malignity, $\mathrm{n}(\%)$ & $19(5.1)$ & $9(10.3)$ & $103.5)$ & $\mathbf{0 . 0 1 2}$ \\
\hline Chronic kidney disease, $\mathrm{n}(\%)$ & $13(3.5)$ & $6(6.8)$ & $7(2.4)$ & 0.051 \\
\hline Hydroxychloroquine, $\mathrm{n}(\%)$ & $80(21.6)$ & $17(19.5)$ & $63(22.3)$ & 0.581 \\
\hline Favipiravir, $\mathrm{n}(\%)$ & $215(58.3)$ & $51(58.6)$ & $164(58.1)$ & 0.939 \\
\hline $\begin{array}{l}\text { Hydroxychloroquine and favipiravir } \\
\text { together, } \mathrm{n}(\%)\end{array}$ & $74(20.1)$ & $19(21.8)$ & $55(19.5)$ & 0.635 \\
\hline Existence of pneumonia, $\mathrm{n}(\%)^{\dagger}$ & $279(75.6)$ & $77(88.5)$ & $202(71.6)$ & $\mathbf{0 . 0 0 1}$ \\
\hline
\end{tabular}

*Median and interquartile range (IQR); ${ }^{\dagger}$ At first admission to hospital. Age and length of hospitalization variables were tested using Student's $t$-test, existence of chronic obstructive pulmonary disease, malignity, and chronic kidney disease variable were tested using Fisher's exact test, and all other variables (i.e., gender, age above 65 years, existence of hypertension, diabetes mellitus, coronary artery disease, pneumonia, hydroxychloroquine, favipiravir, hydroxychloroquine, and favipiravir together) were tested using chi-square test. Bold numbers indicate the significant $p<0.05$. 
Table 2. Laboratory test results of patients with COVID-19 with or without 30-day readmission to hospital.

\begin{tabular}{|c|c|c|c|c|}
\hline \multirow[b]{2}{*}{ Laboratory results } & \multicolumn{3}{|c|}{ Mean \pm standard deviation } & \multirow[b]{2}{*}{ p-value } \\
\hline & $\begin{array}{l}\text { Total participants } \\
\qquad(\mathrm{n}=369)\end{array}$ & $\begin{array}{l}\text { With readmission } \\
\qquad(\mathrm{n}=87)\end{array}$ & $\begin{array}{l}\text { Without readmission } \\
\qquad(\mathrm{n}=282)\end{array}$ & \\
\hline Alanine aminotransferase (IU/L) & $30.70 \pm 35.68$ & $33.84 \pm 36.43$ & $29.71 \pm 35.45$ & 0.348 \\
\hline Aspartate aminotransferase (IU/L) & $32.11 \pm 26.11$ & $37.35 \pm 36.65$ & $30.39 \pm 21.40$ & 0.033 \\
\hline Lactate dehydrogenase (IU/L) & $266.49 \pm 210.93$ & $327.06 \pm 385.08$ & $248.02 \pm 108.73$ & 0.003 \\
\hline C-reactive protein (mg/L) & $520.71 \pm 670.04$ & $700.82 \pm 84.83$ & $470.12 \pm 59.59$ & 0.004 \\
\hline Ferritin $(\mu \mathrm{g} / \mathrm{L})$ & $370.65 \pm 420.03$ & $450.45 \pm 499.71$ & $343.82 \pm 387.17$ & 0.055 \\
\hline D-Dimer (nmol/L) & $4160.94 \pm 7255.43$ & $5985.32 \pm 9667.93$ & $3594.56 \pm 6236.89$ & 0.007 \\
\hline Creatinine $(\mu \mathrm{mol} / \mathrm{L})$ & $82.21 \pm 50.39$ & $1.02 \pm 0.59$ & $0.91 \pm 0.56$ & 0.115 \\
\hline Hemoglobin (g/L) & $130.19 \pm 10.97$ & $13.35 \pm 2$ & $13.14 \pm 1.96$ & 0.375 \\
\hline Leukocytes (×10\%/L) & $6.96 \pm 3.92$ & $8.02 \pm 5.65$ & $6.64 \pm 3.14$ & 0.004 \\
\hline Platelet count $\left(\times 10^{9} / \mathrm{L}\right)$ & $220.80 \pm 81.87$ & $213.95 \pm 90.86$ & $222.92 \pm 78.94$ & 0.373 \\
\hline Neutrophil count $\left(\times 10^{9} / L\right)$ & $4.90 \pm 0.34$ & $5.79 \pm 4.57$ & $4.64 \pm 2.92$ & 0.006 \\
\hline Lymphocyte count (×109/L) & $1.45 \pm 1.53$ & $1.50 \pm 2.83$ & $1.42 \pm 0.79$ & 0.681 \\
\hline $\begin{array}{l}\text { Neutrophil to lymphocyte } \\
\text { count ratio }\end{array}$ & $5.07 \pm 5.90$ & $6.43 \pm 6.24$ & $4.64 \pm 5.74$ & 0.013 \\
\hline
\end{tabular}

Alanine aminotransferase, aspartate aminotransferase, ferritin, D-dimer and lymphocyte count were tested using Mann-Whitney $U$ test, and any other parameters (i.e., lactate dehydrogenase, C-reactive protein, creatinine, hemoglobin, leukocyte, platelet count, neutrophil cunt, lymphocyte count and neutrophil to lymphocyte count ratio) were tested using Student's $t$-test. Bold numbers indicate the significant $p<0.05$.

Table 3. Logistic regression analysis results for readmission of patients with COVID-19.

\begin{tabular}{l|c|c} 
Characteristics & $\begin{array}{c}\text { Readmission odds } \\
\text { ratio }(95 \% \mathrm{Cl})\end{array}$ & $\mathrm{p}$-value \\
\hline Malignity & $3.45(1.19-10.01)$ & $\mathbf{0 . 0 2 2}$ \\
\hline $\begin{array}{l}\text { Existence of pneumonia } \\
\text { at first admission }\end{array}$ & $2.81(1.37-5.75)$ & $\mathbf{0 . 0 0 5}$ \\
\hline Male/female & $1.01(0.58-1.76)$ & 0.959 \\
\hline Hemoglobin (g/L) & $0.90(0.77-1.04)$ & 0.152 \\
\hline $\begin{array}{l}\text { Length of } \\
\text { hospitalization }\end{array}$ & $0.96(0.91-1.02)$ & 0.187 \\
\hline
\end{tabular}

Cl: confidence interval. Bold numbers indicate the significant $p<0.05$.

\section{DISCUSSION}

A total of 369 discharged patients who had treatment for COVID-19 were inspected in this study. Of note, 87 (23.5\%) patients returned to the hospital with symptoms related to COVID-19 within 30 days of discharge. Nine (2.4\%) of these patients were readmitted to the hospital and only one $(0.2 \%)$ died. Existence of pneumonia, lengthening of hospitalization period in the first admission, and higher AST, LDH, CRP values, leukocyte and neutrophil counts, and NLR on the first day of hospital admission were found to be related to increased return risk to the hospital after discharge.

In a study reported from the United States, 275 patients who were confirmed with COVID-19 using PCR test were inspected, $21(7.6 \%)$ of these patients were readmitted to hospital and $6(2.2 \%)$ of them died in 30 days after discharge from the hospital ${ }^{10}$. In another study from the United States, 151 discharged patients with COVID-19 were inspected, and 36 (24.0\%) of them were readmitted to the hospital within 30 days of discharge; about half of these patients were readmitted with complaints related to COVID-19, but the other half were readmitted with trauma, conditions related to hematological, oncological, and neurological diseases. Sixteen (11\%) of these patients were readmitted to hospital ${ }^{11}$. In a study with 1344 patients, after discharge from the hospital, $216(16.5 \%)$ patients were reported to return to the hospital for reasons related to COVID-19, $132(9.8 \%)$ were readmitted to the hospital, and $32(2.4 \%)$ of these died in hospital after readmission $^{3}$. In this reported study, return rates were relatively higher but readmissions to the hospital were lower. Since COVID-19 is a mysterious disease that is new to the population, perception and anxiety levels for this disease are higher than expected, and thus, patients may feel 
a more serious disease period. In a study, 2897 unconfirmed cases of influenza were inspected, and 409 (14\%) of these patients were readmitted to the hospital within 30 days of discharge ${ }^{12}$.

When compared with the data of this reported study, these rates are lower. This may be explained by the increased anxiety and perception of COVID-19 as a dangerous disease in the population.

A study with more than 29,000 patients who had confirmed COVID-19 reported the length of hospitalization time as 8 days ${ }^{13}$. A Korean study reported the length of hospitalization due to COVID-19 as 17 days $^{14}$. In this study, the mean length of stay for hospitalization for COVID-19 was 6 days. Return rates for patients who stayed in the hospital for more than 6 days were higher. Differences in the length of hospitalization periods are thought to be resulting from different treatments and follow-up recommendations varying from country to country. In addition, patients who had more serious COVID-19 disease course were hospitalized for an increased period, and these patients are expected to have more frequent complaints and return rates to the hospital related to COVID-19 within 30 days of discharge.

Concomitant diseases were found to be more frequent in patients with COVID-19 who were readmitted to the hospital after discharge in a study ${ }^{15}$. A total of 154 hospitalized patients who were tested positive using PCR test and who were probable cases (patients who tested negative but had high clinical suspicion of COVID-19) were inspected in a study; patients who were readmitted to the hospital with COVID-19-related symptoms within 30 days of discharge were compared with patients who did not. The readmission rate of patients who had malignancies was found higher ${ }^{5}$. Another study reported that the most frequent concomitant disease in readmitted patients with COVID-19 was hypertension ${ }^{16}$. In this reported study, hypertension was also the most frequent concomitant disease in patients returning to the hospital, but when considering comorbidities, the existence of malignancies was found to be the only risk factor for returning to the hospital within 30 days of discharge.

Dyspnea was reported to be the most frequent returning symptom to the hospital that was observed nearly in half of the patients ${ }^{7}$. The most frequent readmission symptom was dyspnea in another study with 1,062 patients ${ }^{2}$. In this reported study, dyspnea was also the most frequent returning symptom that was explained by the high rate of the existence of pneumonia on the first admission.

In a study with 1,368 patients with COVID-19, the return rate of patients who had higher NLR and D-dimer levels was reported to be higher ${ }^{6}$. This reported study reveals that AST, LDH, CRP levels, leukocyte counts, neutrophil counts, and NLR were higher in patients who returned to the hospital with symptoms related to COVID-19, concordantly. These parameters are usually found elevated in patients with COVID-19 and levels increased as a reflection of the severity of the disease.

This study has limitations. The setup was retrospective, and it was performed in a single center and may not reflect the general population. The relationship of symptoms that resulted in the patient's return to the hospital with other reasons other than COVID-19 was not inspected in detail.

\section{CONCLUSIONS}

The returning rate of patients with COVID-19 within 30 days of discharge was found to be increased in patients who had pneumonia in the first admission and who had increases in inflammatory markers such as leukocyte counts and CRP. In addition, patients with increased D-dimer levels, prolonged stay in the hospital, and patients who had concomitant malignancies were more frequently expected to return to the hospital after discharge. These factors will negatively be affecting morbidity rates, and thus, these patients are more likely to have unplanned hospital visits. Although the return rate within 30 days of discharge was high in this study, readmission to hospital and mortality rates were low. Since COVID-19 is a new and enigmatic disease and its long-term effects are not yet elucidated, it may be wise to recommend following these patients for a more prolonged period, from 6 months to 1 year after discharge from hospital.

\section{ACKNOWLEDGMENT}

The authors thank Prof. Dr. Bulent A. Besirbellioglu and Prof. Dr. M. Metin Yildirimkaya for advice, and Mr. Fatih Acikgoz for statistical analysis and advice.

\section{AUTHORS" CONTRIBUTIONS}

RA: Conceptualization, Data curation, Formal analysis, Investigation, Methodology, Project administration, Resources, Software, Supervision, Validation, Visualization, Writing - original draft, Writing - review \& editing. MBK: Data curation, Investigation, Methodology, Resources, Software, Supervision, Visualization, Writing - original draft, Writing - review \& editing. KSY: Data curation, Investigation, Methodology, Resources, Supervision, Writing - review \& editing. 


\section{REFERENCES}

1. Ministry of Health. COVID-19 Information Platform. [cited on July 12, 2021]. Available from: https://covid19.saglik.gov.tr/ TR-66935/genel-koronavirus-tablosu.html.

2. Yeo I, Baek S, Kim J, Elshakh H, Voronina A, Lou MS, et al. Assessment of thirty-day readmission rate, timing, causes and predictors after hospitalization with COVID-19. J Intern Med. 2021;290(1):157-65. https://doi.org/10.1111/joim.13241

3. Kingery JR, Martin PBF, Baer BR, Pinheiro LC, Rajan M, Clermont A, et al. Thirty-day post-discharge outcomes following COVID-19 infection. J Gen Intern Med. 2021;36(8):2378-85. https://doi. org/10.1007/s11606-021-06924-0

4. Ayoubkhani D, Khunti K, Nafilyan V, Maddox T, Humberstone $B$, Diamond I, et al. Post-covid syndrome in individuals admitted to hospital with covid-19: retrospective cohort study. BMJ. 2021;372:n693. https://doi.org/10.1136/bmj.n693

5. Uyaroğlu OA, Başaran NC, Özışik L, Dizman GT, Eroğlu I, Şahin TK, et al. Thirty-day readmission rate of COVID-19 patients discharged from a tertiary care university hospital in Turkey: an observational, single-center study. Int J Qual Health Care. 2021;33(1):mzaa144. https://doi.org/10.1093/intqh//mzaa144

6. Parra LM, Cantero M, Morrás I, Vallejo A, Diego I, JiménezTejero $\mathrm{E}$, et al. Hospital readmission of discharged patients with COVID-19. Int J Gen Med. 2020;13:1359-66. https:// doi.org/10.2147/IJGM.S275775

7. Somani S, Richter F, Fuster V, De Freitas J, Naik N, Sigel K, et al. Characterization of patients who return to hospital following discharge from hospitalization for COVID19. medRxiv. 2020;2020.05.17.20104604. https://doi. org/10.1101/2020.05.17.20104604

8. Huang $C$, Huang L, Wang $Y$, Li X, Ren L, Gu X, et al. 6-month consequences of COVID-19 in patients discharged from hospital: a cohort study. Lancet. 2021;397(10270):220-32. https://doi.org/10.1016/S0140-6736(20)32656-8
9. Turkey. Ministry of Health. COVID-19 (SARS-CoV-2 infection). [cited on May 5, 2021]. Available from: https://covid19.saglik. gov.tr/Eklenti/40719/0/covid-19rehberieriskinhastayonetimiv etedavipdf.pdf.

10. Guarin G, Lo KB, Bhargav R, Salacup G, Wattoo A, Coignet $J G$, et al. Factors associated with hospital readmissions among patients with COVID-19: a single-center experience. J Med Virol. 2021;93(9):5582-7. https://doi.org/10.1002/jmv.27104

11. Gwin M, Saleki M, Lampert H, Meo N, Bann M. Emergency department visits and readmissions after COVID-19 hospitalization: a cross-sectional analysis. Intern Emerg Med. 2021;16(6):1715-8. https://doi.org/10.1007/s11739021-02644-9

12. Dobrzynski DM, Ndi DN, Zhu Y, Markus T, Schaffner W, Talbot HK. Hospital readmissions after laboratory-confirmed influenza hospitalization. J Infect Dis. 2020;222(4):583-9. https://doi. org/10.1093/infdis/jiaa117

13. Verna EC, Landis C, Brown Jr RS, Mospan AR, Crawford JM, Hildebrand JS, et al. Factors associated with readmission in the US following hospitalization with COVID-19. Clin Infect Dis. 2021:ciab464. https://doi.org/10.1093/cid/ciab464

14. Jeon $\mathrm{WH}$, Seon JY, Park SY, Oh IH. Analysis of risk factors on readmission cases of COVID-19 in the Republic of Korea: using nationwide health claims data. Int J Environ Res Public Health. 2020;17(16):5844. https://doi.org/10.3390/ijerph17165844

15. Atalla E, Kalligeros M, Giampaolo G, Mylona EK, Shehadeh F, Mylonakis E. Readmissions among patients with COVID-19. Int J Clin Pract. 2021;75(3):e13700. https://doi.org/10.1111/ ijcp. 13700

16. Durmus E, Guneysu F. Evaluation of re-hospitalized COVID-19 patients in a hospital. Rev Assoc Med Bras (1992). 2020;66(12):1690-5. https://doi.org/10.1590/18069282.66.12.1690 